\title{
Suicidal ideation and associated factors among school-going adolescents in rural Uganda Emmanuel Rudatsikira1 ${ }^{1}$, Adamson S Muula*2, Seter Siziya ${ }^{3}$ and Jeremiahs Twa-Twa ${ }^{4}$
}

Address: ${ }^{1}$ Departments of Biostatistics and Epidemiology and Global Health, Loma Linda University, School of Public Health, Loma Linda, California, USA, ${ }^{2}$ Department of Community Health, University of Malawi, College of Medicine, Blantyre, Malawi, ${ }^{3}$ Department of Community Medicine, University of Zambia, School of Medicine, Lusaka, Zambia and ${ }^{4}$ Principal Medical Officer, Child Health Division, Ministry of Health, Kampala, Uganda

Email: Emmanuel Rudatsikira - erudatsikira@llu.edu; Adamson S Muula* - muula@email.unc.edu; Seter Siziya - ssiziya@yahoo.com; Jeremiahs Twa-Twa - twatwaj@yahoo.com

* Corresponding author

Published: 23 November 2007

BMC Psychiatry 2007, 7:67 doi:10.1 186/147|-244X-7-67
Received: 30 April 2007

Accepted: 23 November 2007

This article is available from: http://www.biomedcentral.com/I47/-244X/7/67

(C) 2007 Rudatsikira et al; licensee BioMed Central Ltd.

This is an Open Access article distributed under the terms of the Creative Commons Attribution License (http://creativecommons.org/licenses/by/2.0), which permits unrestricted use, distribution, and reproduction in any medium, provided the original work is properly cited.

\begin{abstract}
Background: Mental health is a neglected area of health research and practice in most of subSaharan African countries where the largest burden of morbidity is from infectious diseases. This even occurs despite the fact that some mental health problems may arise from infectious diseases.

Methods: We conducted secondary analysis of the Uganda Global School-Based Health Survey2003 to obtain the prevalence of, and assess factors that may be associated with suicidal ideation among school-going adolescents in rural Uganda. Assessment of association was conducted through both bi-variate and multivariate logistic regression analysis.

Results: Altogether $21.6 \%$ of the study participants, $21.3 \%$ males and $23.5 \%$ females had seriously considered committing suicide within the past 12 months. Loneliness, worry were positively associated with suicide ideation after adjusting for age, gender, smoking, drinking, and experience of having been bullied $(\mathrm{OR}=1.59 ; 95 \% \mathrm{Cl}[1.12,2.26]$ and $\mathrm{OR}=1.19 ; 95 \% \mathrm{Cl}[1.12,2.25])$ respectively. Males were less likely to seriously consider committing suicide than females $(O R=$ $0.70 ; 95 \% \mathrm{Cl}[0.50,0.98])$.

Conclusion: Adolescent suicidal ideation is a major public health issue in rural Uganda. Measures aimed to prevent adolescent suicides in Uganda should incorporate our understanding of factors that are associated with suicide in rural Uganda such the gender disparity and the association observed with substance use.
\end{abstract}

\section{Background}

Globally, it is estimated that there were 786,000 suicide deaths in 1990 [1]. These deaths were only slightly lower than the number of deaths $(900,000)$ estimated for lung cancer in 1990 [2]. Yet lung cancer has received attention among health care professionals and the general public while suicide has largely not attracted that much attention. There is paucity of data on suicidal ideation, attempted suicide and suicide among adolescents in Africa. This dearth of information is likely to be multi-fac- 
torial in origin, including in part the global lack of adequate interest in mental health research in general and limited funding. Many of the health systems in southern Africa are engaged more in the prevention and treatment of communicable diseases and not mental health issues. Even in developed nations, suicide among children has been a neglected issue until recently $[3,4]$. In the few setting were studies have been conducted, suicidal thoughts or ideation has been described as. Among Viennese student for instance, 37.9 percent reported having ever had suicidal thoughts in their lives [5]. Weissman et al. have assessed the lifetime prevalence for suicide ideation from nine countries [6]. This ranged from from 2.09\% (Beirut) to $18.51 \%$ (Christchurch, New Zealand).

Ovuga et al.[7] have reported on lifetime prevalence of suicidal ideation among first year students admitted to Makerere University, Uganda. Among this sample, lifetime prevalence was $56.0 \%$ among first years student in disciplines other than medicine while prevalence was only $8.9 \%$ among first year medical students. We are unaware of previous research on suicidal ideation among inschool adolescents in rural Uganda. Our study was aimed to assess the prevalence and associated factors of suicidal ideation among school going adolescents in rural Uganda. We believe information obtained may the design, implementation and evaluation of public health intervention aimed to prevent suicides in Uganda.

\section{Methods}

Our study was a secondary analysis of existing data available from the Uganda Global School-Based Health Survey (GSHS) conducted in 2003. The GSHS was developed by the World Health Organization (WHO) in collaboration with United Nations' UNICEF, UNESCO, and UNAIDS with technical assistance from Centers for Diseases Control and Prevention (CDC, United States). The GSHS aims to provide data on health and social behaviours among school-going adolescent students.

Variables that were used for our analysis included sociodemographic characteristics, having considered suicide in the past 12 months, tobacco cigarette and cannabis smoking, alcohol use, having been worried so as to affect sleep and parental supervision. The questions on suicide, worry and loneliness were: During the past 12 months, did you ever seriously consider attempting suicide? During the past 12 months, how often have you been so worried about something that you could not sleep at night? During the past 12 months, have you felt lonely?

The GSHS uses a two-stage probability sampling technique, in which for the first stage, primary sampling units are schools which are selected with a probability proportional to their enrolment size. In the second step a system- atic sample of classes in the selected school are obtained. All students in the selected classes are eligible to participate. A self-completed questionnaire is used.

For the rural Uganda survey, overall response rate was 67 percent. Data analysis was performed using SUDAAN software version 9.0.1 (Research Triangle Institute, Research Triangle Park, North Carolina, United States of America). Our analysis was based on compete case analysis as no imputation of missing observations was carried out. We obtained frequencies of attributes. A weighting factor was used in the analysis to obtain prevalence estimates so as to reflect the likelihood of sampling each student and to reduce bias by compensating for differing patterns of non response. The weight used for estimation is given by the following formula:

$$
\mathrm{W}=\mathrm{W} 11^{*} \mathrm{~W} 2 * \mathrm{f} 1 * \mathrm{f} 2 * \mathrm{f} 3 * \mathrm{f} 4
$$

where $\mathrm{W} 1$ = the inverse of the probability of selecting the school

$\mathrm{W} 2$ = the inverse of the probability of selecting the classroom within the school

$\mathrm{f} 1$ = a school-level non response adjustment factor calculated by school size category (small, medium, large)

$\mathrm{f} 2$ = a class-level non response adjustment factor calculated for each school

f3 = a student-level non response adjustment factor calculated by class

$\mathrm{f} 4$ = a post stratification adjustment factor calculated by grade.

We also conducted logistic regression analysis to estimate the association between suicidal thoughts and relevant predictor variables. We report unadjusted bi-variate analysis between suicidal thoughts and a predictor variable. We thereafter report results for adjusted odds ratios for the factors.

\section{Ethical considerations}

A committee with members drawn from the Ministry of Education and Ministry of Health oversaw data collection. The study protocol was reviewed and approved by the National Council for Science and Technology of Uganda. Permission to conduct the study was granted by the Headmasters of the respective schools. All eligible students were requested to complete an anonymous questionnaire. However, students were free to participate or not to participate. Non participation could either be overt, such as 
clearly indicating that they would not like to participate or by submitting a non-completed questionnaire.

\section{Results}

Table 1 presents selected characteristics of the study population of 1,506 Ugandan school going adolescents aged 11 to 17 years (median 15 years old) who participated in the study. Most of the sample was males (53.3\%) and 1617 years old $(40.4 \%)$. The majority reported that parents supervised them when out of school $(63.3 \%)$. One in 2 respondents had been bullied at least once in the last 30 days $(52.3 \%), 5.7 \%$ were smokers, $17.7 \%$ were alcohol drinkers. $21.6 \%$ of the study participants, $21.3 \%$ males and $23.5 \%$ females had seriously considered committing suicide within the past 12 months.

Table 2 indicates that male subjects were less likely to contemplate committing suicide than females $(\mathrm{OR}=0.71$; $95 \%$ CI $[0.55,0.93])$. Feelings of loneliness were positively associated with suicide ideation for both males and females $(\mathrm{OR}=1.60 ; 95 \% \mathrm{CI}[1.03,2.33]$ and $\mathrm{OR}=2.09$;

Table I: Socio-demographic and behavioral characteristics of study participants in the Global School Health Sample for rural Uganda, 2003

\begin{tabular}{|c|c|c|c|}
\hline & $\begin{array}{l}\text { Total } \\
N=1,506\end{array}$ & $\begin{array}{l}\text { Males } \\
N=784\end{array}$ & $\begin{array}{l}\text { Females } \\
N=676\end{array}$ \\
\hline & $\%(n)$ & $\%(n)$ & $\%(n)$ \\
\hline \multicolumn{4}{|l|}{ Age (years) } \\
\hline $11-13$ & $13.4(192)$ & $10.2(86)$ & $14.3(97)$ \\
\hline 14 & $21.0(30 \mathrm{I})$ & $15.9(126)$ & $24.0(168)$ \\
\hline 15 & $29.4(422)$ & $28.6(227)$ & $26.7(181)$ \\
\hline $16-17$ & $36.2(519)$ & $45.2(345)$ & $35.0(230)$ \\
\hline \multicolumn{4}{|l|}{ Gender } \\
\hline Females & $46.3(676)$ & - & - \\
\hline Males & $53.7(784)$ & - & - \\
\hline \multicolumn{4}{|l|}{ Worry } \\
\hline No & $54.5(808)$ & $25.1(200)$ & $24.3(163)$ \\
\hline Yes & $45.6(676)$ & $74.9(584)$ & $75.7(5 \mid 3)$ \\
\hline \multicolumn{4}{|l|}{ Loneliness } \\
\hline No & $58.7(857)$ & $35.5(264)$ & $28.5(190)$ \\
\hline Yes & $41.3(603)$ & $64.5(520)$ & $71.5(486)$ \\
\hline \multicolumn{4}{|c|}{ Cigarette smoking } \\
\hline No & $94.3(|3| \mid)$ & $92.1(691)$ & $96.8(620)$ \\
\hline Yes & $5.7(74)$ & $7.9(55)$ & $3.2(19)$ \\
\hline \multicolumn{4}{|l|}{ Drinking } \\
\hline No & $82.3(|07|)$ & $73.4(553)$ & $50.8(5 \mid 8)$ \\
\hline Yes & $17.7(230)$ & $26.6(200)$ & $49.2(501)$ \\
\hline \multicolumn{4}{|c|}{ Parental supervision } \\
\hline No & $36.7(537)$ & $36.6(286)$ & $36.7(25 \mathrm{I})$ \\
\hline Yes & $63.3(923)$ & $63.4(496)$ & $63.3(425)$ \\
\hline \multicolumn{4}{|l|}{ Bullied } \\
\hline No & $47.7(610)$ & $44.7(306)$ & $51.1(304)$ \\
\hline Yes & $52.3(652)$ & $55.3(369)$ & $48.9(283)$ \\
\hline \multicolumn{4}{|c|}{ Seriously considered suicide } \\
\hline No & $78.0(1066)$ & 78.7 (539) & $76.5(50 I)$ \\
\hline Yes & $22.0(300)$ & $21.3(146)$ & $23.5(154)$ \\
\hline
\end{tabular}

Note: Missing values were not included in the calculation of percentages
95\% CI [1.43, 3.03]). Worry and alcohol drinking were positively associated with suicide ideation among females only $(\mathrm{OR}=2.42 ; 95 \% \mathrm{CI}[1.66,3.54]$ and $\mathrm{OR}=1.81 ; 95 \%$ $[1.05,3.13])$. For both males and females, being bullied was positively associated with suicide ideation $(\mathrm{OR}=$ $1.62 ; 95 \% \mathrm{CI}[1.06,2.47]$ and $\mathrm{OR}=1.63 ; 95 \% \mathrm{CI}[1.08$, 2.46]).

Table 3 presents results from multivariate analysis. Worry and loneliness remained positively associated with suicide ideation after adjusting for age, gender, cigarettes smoking, alcohol drinking and experience of having been bullied (OR $=1.19 ; 95 \%$ CI $[1.12,2.25]$ and $\mathrm{OR}=1.59$; $95 \%$ CI $[1.12,2.26])$. Likewise, male gender remained negatively associated with suicide ideation in multivariate analysis $(\mathrm{OR}=0.70 ; 95 \% \mathrm{CI}[0.50,0.98])$.

\section{Discussion}

21.6 percent of the study participants, $21.3 \%$ males and $23.5 \%$ females had seriously considered committing suicide within the past 12 months. Factors positively associated with a history of suicidal ideation were female gender, being victim of bullying, lack of parental supervision, current cigarette smoking, and alcohol use. All of these factors have been described as being associated with suicidal ideation in other studies [8-12]. Thirty-six percent of the general population has reported history of suicidal ideation Uganda [13]. The estimate from Uganda is much lower than the $39.7 \%$ life time prevalence among Viennese students [5]. However our study looked at 12 months period and so comparisons between the two settings may not be reasonable. Due to the design of our study, we are unable to ascribe causation i.e. it is not possible to say these factors are causes of suicidal ideation.

Over half $(52.3 \%)$ of the study participants in rural Uganda reported being victims of bullying. This prevalence estimate is much higher than 19.3\% found among school going adolescents in Durban and Cape Town in South Africa [14]. In the Uganda study, participants were asked the question: During the past 30 days, on how many days were you bullied? In the South African study, students were asked: During the past 12 months, have you been bullied at school? Even though the Uganda study asked for a much shorter period (30 days compared to 12 months in South Africa), the Uganda survey was associated with a much higher estimate. We are unable to specifically identify the reasons for these differences. A possibility is that indeed adolescents in rural Uganda are more likely to be bullied than in South Africa. The second possibility is the way the questions were asked. In South Africa, the questions aimed to identify bullying occurring at school. In the Uganda study, this was not specified and study participants may have reported bullying occurring in and out of school. Despite these differences, the preva- 
Table 2: Bivariate Logistic regression results between having considered suicide and predictor variables

\begin{tabular}{|c|c|c|c|}
\hline & Total & Males & Females \\
\hline \multicolumn{4}{|l|}{ Age (years) } \\
\hline $11-13$ & 1.00 & 1.00 & 1.00 \\
\hline 14 & $1.12[0.68,1.86]$ & $1.51[0.64,3.59]$ & $0.96[0.17,1.81]$ \\
\hline 15 & $1.23[0.77,1.98]$ & $1.14[0.5 \mathrm{I}, 2.53]$ & $1.43[0.48,1.75]$ \\
\hline $16-17$ & $1.23[0.78,1.95]$ & $1.77[0.82,3.79]$ & $0.96[0.52,1.76]$ \\
\hline \multicolumn{4}{|l|}{ Gender } \\
\hline Females & 1.00 & - & - \\
\hline Males & $0.71[0.55,0.93]$ & - & - \\
\hline \multicolumn{4}{|l|}{ Worry } \\
\hline No & 1.00 & 1.00 & 1.00 \\
\hline Yes & $1.71[1.31,2.22]$ & $1.25[0.86,1.82]$ & $2.42[1.66,3.54]$ \\
\hline \multicolumn{4}{|l|}{ Loneliness } \\
\hline No & 1.00 & 1.00 & 1.00 \\
\hline Yes & $1.79[1.37,2.32]$ & $1.60[1.03,2.33]$ & $2.09[1.43,3.04]$ \\
\hline \multicolumn{4}{|c|}{ Cigarette smoking } \\
\hline No & 1.00 & 1.00 & 1.00 \\
\hline Yes & $1.75[1.01,3.04]$ & $1.59[0.81,3.12]$ & $2.35[0.74,7.45]$ \\
\hline \multicolumn{4}{|l|}{ Drinking } \\
\hline No & 1.00 & 1.00 & 1.00 \\
\hline Yes & $1.40[0.99,2.00]$ & $1.12[0.68,1.85]$ & $1.81[1.05,3.13]$ \\
\hline \multicolumn{4}{|c|}{ Parental supervision } \\
\hline No & 1.00 & 1.00 & 1.00 \\
\hline Yes & $0.87[0.66,1.13]$ & $0.93[0.63,1.36]$ & $0.81[0.55,1.18]$ \\
\hline \multicolumn{4}{|l|}{ Bullied } \\
\hline No & 1.00 & 1.00 & 1.00 \\
\hline Yes & $1.58[1.18,2.11]$ & $1.62[1.06,2.47]$ & $1.63[1.08,2.46]$ \\
\hline
\end{tabular}

Table 3: Multivariate Logistic regression results between having considered suicide and predictor variables

\begin{tabular}{ll}
\hline Age (years) & 1.00 \\
$11-13$ & $0.91[0.49,1.70]$ \\
14 & $0.90[0.50,1.63]$ \\
15 & $1.15[0.66,2.00]$ \\
$16-17$ & 1.00 \\
Gender & $0.70[0.50,0.98]$ \\
Females & \\
Males & 1.00 \\
Loneliness & $1.59[1.12,2.26]$ \\
No & \\
Yes & 1.00 \\
Worry & $1.19[1.12,2.25]$ \\
No & \\
Yes & 1.00 \\
Cigarette smoking & $1.29[0.55,3.01]$ \\
No & \\
Yes & 1.00 \\
Drinking & $1.27[0.80,2.01]$ \\
No & 1.00 \\
Yes & $1.34[0.96,1.87]$ \\
Bullied & \\
No & \\
Yes &
\end{tabular}

*Adjustments made for age, gender, loneliness, worry and cigarette smoking. lence of having been bullied in the past 30 days appears to be unacceptably high in rural Uganda.

Current cigarette smoker (5.7\%) was significantly lower than the $21.9 \%$ reported by Mpabulungi and Muula for Arua, rural Uganda for 2002 [15]. While in our study cigarette smoking is for a representative sample of all rural Uganda, Mpabulungi and Muula estimates were for one district, which was also tobacco growing. Bolton et al have reported that use of alcohol and cigarette smoking may be initiated or maintained as self-medications among persons who have anxiety disorders, in themselves risk factors for suicide [16]. Liu et al have reported increased prevalence of suicidal ideation with age among youths in rural China [17]. We also found that the odds of suicidal ideation increased with increasing age.

Studies from other settings have reported female over-representation among participants reporting suicidal ideation has been reported elsewhere [8-10]. In our study however, the gender disparity was minimal i.e. $21.3 \%$ and $23.5 \%$ among males and females.

Females may also be over-represented among individuals who actually attempt or commit suicide $[18,19]$. In a facility based study of 45 suicide and attempted suicide 
patients in rural Namibia, Ikealumba and Couper reported that 23 were female while 21 were males [20].

While the gender disparity in suicidal ideation in many settings is that females are more likely to have suicidal thoughts, the gender disparity is reversed with regard to suicide commission i.e. males are more likely to commit suicide and using more violent means than females [21]. Brezo et al.[22] have found that female gender was also associated with a 2-3 times greater likelihood of suicide attempts among Canadian-French participants.

\section{Limitations of this study}

Our study has several limitations. We did not collect data on other factors that may have effect on suicidality. For instance evidence reported in a meta-analysis by Bridge et al suggest that antidepressant drugs may be associated with suicidality [23]. Other studies have also demonstrated that psychiatric co-morbidity is an important proximal determinant of suicidal behaviors [24-26]. These other factors were not assessed largely because we used secondary data. The study also recruited participants from school-going adolescents. To the extent that school-going adolescents are different in their prevalence and factors associated with suicidal ideation, our study may not be representative of all adolescents in rural Uganda. It is also important to recognize that not all suicidal ideation will result in suicide attempt or suicide [21]. However, adolescents who have suicidal thoughts are more likely to attempt or commit suicidal compared to those without the thoughts. Brezo et al have reported that correlates of suicide attempts in suicidal ideators may vary as a function of the persistence of suicidal ideas and gender [22]. Among Canadian-French study participants, these authors found that persistent suicidal ideas, Axis I psychopathology, female gender and childhood sexual abuse (CSA) were the most consistent correlates of suicide attempts. Externalizing disorders were more important among males.

\section{Conclusion}

Our study has found that the prevalence of suicidal ideation among adolescent students in rural Uganda was $21.6 \%$. Being females, increasing age, cigarette smoking, alcohol, having been bullied, loneliness, significant worry and lack of parental supervision were associated with suicidal ideation. There is need to recognize suicidal ideation as an important issue among adolescents in rural Uganda. The design, implementation and evaluation of public health interventions aimed at reducing suicide in Uganda should incorporate our understanding of the factors associated with suicide. Ovuga et al.[27] have described how mental health care leaders in Uganda are currently promoting the provision of care where for decades since independence, mental health has been largely neglected. This initiative has potential to mobilize attention and support towards mental health research and care in Uganda.

\section{Abbreviations}

CDC: Centers for Disease Control and Prevention

GSHS: Global School-Based Health Survey

UNAIDS: Joint United Nations Program on HIV/AIDS

UNESCO: United Nations Educational, Scientific and Cultural Organization

UNICEF: United Nations Children's Fund

WHO: World Health Organization

\section{Competing interests}

The author(s) declare that they have no competing interests.

\section{Authors' contributions}

ER conducted the data analysis and participated in drafting of manuscript.

ASM conceived the analysis plan, participated in interpretation of data and drafting of manuscript.

SS participated in the interpretation and drafting of manuscript.

JT coordinated in-country data collection, participated in the interpretation of findings and drafting of manuscript.

All authors approved the final draft of the manuscript.

\section{Acknowledgements}

We thank all adolescents who participated in this study. We also thank the World Health Organization (WHO) and the Centers for Diseases Control and Prevention (CDC) for permission to use the data. However, the WHO and the $C D C$ made do decisions regarding the analysis or decision to publish the findings. ASM is funded through the University of Malawi, College of Medicine Junior Faculty Training Program.

\section{References}

I. Reza A, Mercy JA, Krug E: Epidemiology of violent deaths in the world. Inj Prev 2001, 7: 104-II.

2. Murray CJ, Lopez AD: Mortality by cause for eight regions of the world: Global Burden of Disease Study. Lancet 1997, 349:1269-76.

3. Pomara N: Suicidal behaviour: a neglected issue in DSM-III. J Clin Psychiatry 1984, 45:280.

4. Hammond D, Whittlesey S, Kearns L, Bennett S, Allen SF: Adolescent suicide problems. J Okla State Med Assoc 2004, 97:78-82.

5. Dervic K, Akkaya-Kalayci T, Kapusta ND, Kaya M, Merl E, Vogel E, Pellegrini E, Friedrich $\mathrm{MH}$ : Suicidal ideation among Viennese high school students. Wien Klin Wochesnschr 2007, I 19:174-180.

6. Weissman MM, Bland RC, Canino GJ, Greenwald S, Hwu HG, Joyce PR, Karam EG, Lee CK, Lellouch J, Lepine JP, Newman SC, Rubio-Stipec M, Wells JE, Wickramaratne PJ, Wittchen HU, Yeh EK: Preva- 
lence of suicide ideation and suicide attempts in nine countries. Psychol Med 1999, 29:9-17.

7. Ovuga E, Boardman J, Wasserman D: Undergraduate student mental health at Makerere University, Uganda. World Psychiatry 2006, 5:5I-2.

8. Lam TH, Stewart SM, Yip PS, Leauy GM, Ho LM, Ho SY, Lee PW: Suicidality and cultural values among Hong Kong adolescents. Soc Sci Med 2004, 58:487-98.

9. Borges G, Walters EE, Kessler RC: Associations of substance use, abuse, and dependence with subsequent suicidal behavior. Am J Epidemiol 2000, 151:781-9.

10. McKeown RE, Garrison CZ, Cuffe SP, Waller JL, Jackson KL, Addy $\mathrm{CL}$ : Incidence and predictors of suicidal behaviours in a longitudinal sample of adolescents. J Am Acad Child Adolesc Psychiatry 1998, 37:612-9.

II. Parks HS, Schepp KG, Jang EH, Koo HY: Predictors of suicidal ideation among high school students by gender in South Korea. J Sch Health 2006, 76: 181-8.

12. Ali A, Maharajh HD: Social predictors of suicidal behaviour in Trinidad and Tobago. Soc Psychiatry Psychiatric Epidemiol 2005, 40:186-91.

13. Ovuga E, Boardman J, Wasserman D: The prevalence of depression in two districts of Uganda. Soc Psychiatry Psychiatr Epidemiol 2005, 40:439-45.

14. Liang $\mathrm{H}$, Flisher $\mathrm{AJ}$, Lombard $\mathrm{CJ}$ : Bullying, violence, and risk behaviour in South African school students. Child Abuse Negl 2007, 31 : |6|-7|.

15. Mpabulungi L, Muula AS: Tobacco use among high school students in a remote district of Arua, Uganda. Rural Remote Health 2006, 6:609.

16. Bolton J, Cox B, Clara I, Sareen J: Use of alcohol and drugs to selfmedicate anxiety disorders in a nationally representative sample. J Nerv Ment Dis 2006, 194(I I):8 18-825. 194-8I8-25

17. Liu X, Tein JY, Zhao Z, Sandler IN: Suicidality and correlates among rural adolescents of China. J Adolesc Health 2005, 37:443-51.

18. Meehan SA, Broom Y: Analysis of a national toll free suicide crisis line in South Africa. Suicide and Life Threat Behav 2007, 37:66-78.

19. Lari AR, Joghataei MT, Adli YR, Zadeh YA, Alaghehbandan R: Epidemiology of suicide by burns in the province of Isfahan, Iran. J Burn Care Res 2007, 28(2):307-3II.

20. Ikealumba NV, Couper ID: Suicide and attempted suicide: the Reboboth experience. Rural Remote Health 2006, 6:535.

21. American Academy of Child and Adolescent Psychiatry Association: Practice Parameters for the assessment and treatment of children and adolescents with suicidal behaviours. 2000 [http://www.aacap.org/galleries/PracticeParameters/Suicide.pdf]. Accessed 2 October 2007

22. Brezo J, Paris J, Tremblay R, Vitaro F, Hebert M, Turecki G: Identifying correlates of suicide attempts in suicidal ideators: a population-based study. Psychol Med 2007, 37:155I-I557.

23. Bridge JA, lyengar S, Salary CB, Barbe RP, Birmaher B, Pincus HA, Ren $L$, Brent DA: Clinical response and risk for reported suicidal ideation in pediatric antidepressant treatment: a meta-analysis of controlled trials. JAMA 2007, 297:1683-96.

24. Gould MS, King R, Greenwald S, Fisher P, Schwab-Stone M, Kramer R, Flisher AJ, Goodman S, Canino G, Shaffer D: Psychopathology associated with suicidal ideation and attempts among children and adolescents. J Am Acad Child Adolesc Psychiatry 1998, 37:915-923.

25. Fergusson DM, Lynskey MT: Suicide attempts and suicidal ideation in a birth cohort of 16 -year-old New Zealanders. J Am Acad Child Adolesc Psychiatry 1995, 34:1308-1317.

26. Foley DL, Goldston DB, Costello EJ, Angold A: Proximal psychiatric risk factors for suicidality in youth: the Great Smoky Mountains Study. Arch Gen Psychiatry 2006, 63:1017-24.

27. Ovuga E, Boardman J, Wasserman D: Integrating mental health into primary health care: local initiatives from Uganda. World Psychiatry 2007, 6:60-I.

\section{Pre-publication history}

The pre-publication history for this paper can be accessed here:
http://www.biomedcentral.com/1471-244X/7/67/pre pub
Publish with Bio Med Central and every scientist can read your work free of charge

"BioMed Central will be the most significant development for disseminating the results of biomedical research in our lifetime. "

Sir Paul Nurse, Cancer Research UK

Your research papers will be:

- available free of charge to the entire biomedical community

- peer reviewed and published immediately upon acceptance

- cited in PubMed and archived on PubMed Central

- yours - you keep the copyright 